\title{
Magnetic-field sensor with self-reference characteristic based on a magnetic fluid and independent plasmonic dual resonances
}

\author{
Kun Ren ${ }^{1}$, Xiaobin Ren ${ }^{*}$, Yumeng $\mathrm{He}^{1}$ and Qun Han ${ }^{1}$
}

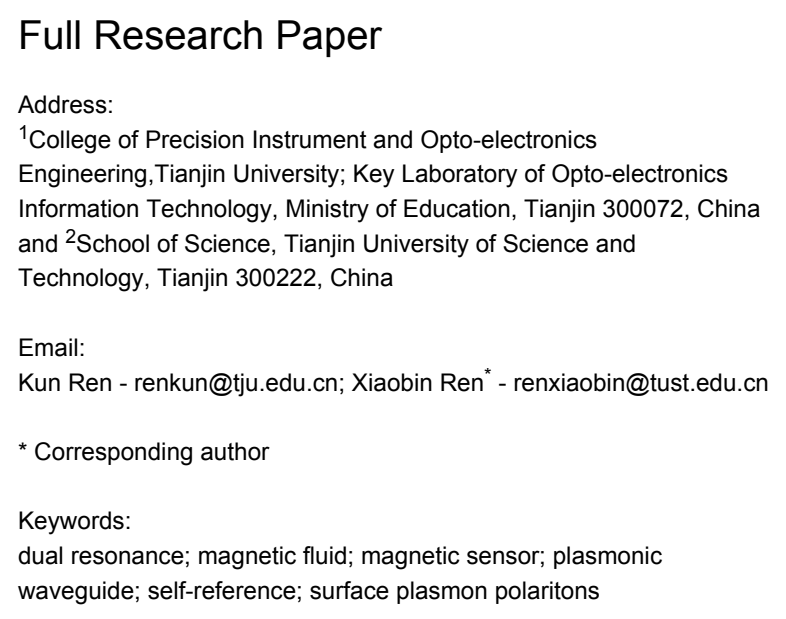

waveguide; self-reference; surface plasmon polaritons

Beilstein J. Nanotechnol. 2019, 10, 247-255. doi:10.3762/bjnano.10.23

Received: 15 October 2018

Accepted: 16 December 2018

Published: 22 January 2019

Associate Editor: N. Motta

(C) 2019 Ren et al.; licensee Beilstein-Institut. License and terms: see end of document.

\begin{abstract}
A magnetic-field sensor with self-reference characteristic based on metal-dielectric-metal (MDM) plasmonic waveguides and a magnetic fluid (MF) is proposed and theoretically investigated. Independent dual resonances are supported by the coupled resonator-waveguide system. The physical mechanisms of dual resonances are analyzed by the temporal coupled-mode theory. The transmission response to an external magnetic field is explored by using the remarkable tunability of the refractive index of the MF. Based on the different dependence of two resonances on the external field, a magnetic-field sensor with self-reference characteristic is achieved. The magnetic-field nanosensor shows an excellent performance with a high sensitivity of $27 \mathrm{pm} / \mathrm{Oe}$, i.e., $270 \mathrm{pm} / \mathrm{mT}$. The proposed sensor takes advantage of the refractive-index tunability of the MF and the compactness of the MDM waveguide structure. This research may open new opportunities to design nanoscale magnetic sensors with good performance.
\end{abstract}

\section{Introduction}

Sensors that can detect the change of environmental conditions are one of the most important devices in optical communication. Optical sensors are obtained by monitoring the change of optical properties based on, e.g., thermo-optic, electro-optic, and magneto-optic effects. In the area of magnetic-field sensors, magnetic fluids (MFs) or ferrofluids have attracted a lot of research interest in recent years [1].
A MF is a stable colloidal suspension of ferromagnetic nanoparticles in certain suitable liquid carriers. It has the remarkable property that the refractive index can be tuned in an applied magnetic field $[2,3]$. The tunability results from the field-induced structural reorganization of suspended magnetic colloidal particles [4]. This field-dependent property of a MF has been utilized to measure magnetic fields and current intensities $[5,6]$. 
Other applications of MFs in optical devices have been proposed, such as tunable optical filters [7], optical switches [8], modulators [9] and optical sensors [10]. In particular, opticalfiber magnetic sensors have been developed by combining MFs with optical-fiber technology [11-15]. Optical-fiber magneticfield sensors have the advantages of easy fabrication and compactness.

In recent years, compact optical devices based on surface plasmon polaritons (SPPs) have been reported. SPPs propagate along the dielectric-metal interface with the amplitudes decaying exponentially into both sides [16]. The deep subwavelength confinement of SPPs leads to the development of various integrated photonic components, such as filters [17], modulators [18], interferometers [19], optical switches [20] and nanosensors [21,22]. As important plasmonic structures, metal-dielectric-metal (MDM) waveguides have attracted considerable attention. Various kinds of plasmonic devices containing MDM waveguides have been investigated [23-27]. MDM waveguide-cavity coupled systems have been reported such as stub cavities [24], side-coupled rectangular cavities [25], T-shape cavities [26] and ring-groove joint cavities [27].

In this paper we propose a compact magnetic-field sensor based on a MF and a plasmonic structure. As far as we know, the combination of a MF with a plasmonic waveguide has not been reported to date. The unique magnetic-optical properties of MFs are the basis of the optical sensor. The plasmonic structure is a MDM waveguide-cavity coupled system that is suitable for chip-scale integration. The proposed sensor takes advantage of the refractive index tunability of MFs as well as of the compactness of the MDM waveguide structure. Furthermore, the proposed magnetic-field sensor has a self-reference characteristic, which can guarantee the detection accuracy. A simple and compact self-reference sensor with high sensitivity is achieved and it is promising in the integrated sensing and detection of magnetic fields.

\section{Methods}

The proposed plasmonic nanostructure is schematically shown in Figure 1. Two stubs are located on each side of the MDM waveguide. A disk resonator is coupled to the upper stub with a coupling distance g. $L_{1}, L_{2}$, and $W_{1}, W_{2}$ denote the length and the width of the stubs, respectively. The radius of disk is $R$ and the width of the MDM waveguide is $W$. The dielectric in the stub and waveguide is water. The disk resonator is filled with magnetic fluid. The background metal in the grey part is silver, the complex permittivity of which is characterized by the well-known Drude model: $\varepsilon_{\mathrm{m}}=\varepsilon_{\infty}-\omega_{\mathrm{p}}^{2} /\left(\omega^{2}+\mathrm{i} \gamma \omega\right)$, where $\varepsilon_{\infty}=3.7$ is the permittivity at infinite angular frequency, the bulk plasma frequency is $\omega_{\mathrm{p}}=1.38 \times 10^{16} \mathrm{~Hz}$, the damping fre- quency of the oscillations is $\gamma=2.73 \times 10^{13} \mathrm{~Hz}$, and $\omega$ is the angular frequency of the incident light.

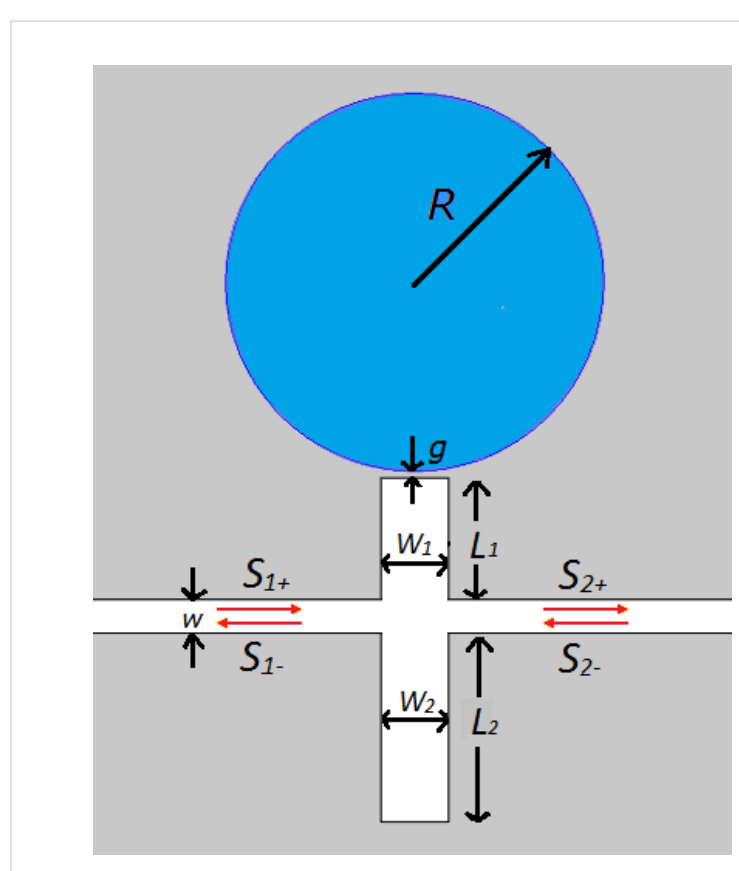

Figure 1: Schematic diagram of the MDM waveguide-resonator structure. The width of the waveguide is $W$ and the radius of the disk is $R$. $L_{1}, L_{2}$, and $W_{1}, W_{2}$ denote the length and the width of two stubs, respectively. The coupling distance between the disk resonator and the upper stub is $g$.

The effective refractive index for SPPs is given by $n_{\text {eff }}=\beta / k_{0}$. The propagation constant $\beta$ can be obtained from the dispersion equation [28]: $\varepsilon_{\mathrm{d}} k_{\mathrm{m}}+\varepsilon_{\mathrm{m}} k_{\mathrm{d}} \tanh \left(k_{\mathrm{d}} w / 2\right)=0$, where $k_{\mathrm{d}}$ and $k_{\mathrm{m}}$ are the transverse propagation constants in the dielectric and the metal, respectively:

$$
k_{\mathrm{d}}=\sqrt{\beta^{2}-k_{0}^{2} \varepsilon_{\mathrm{d}}}, \quad k_{\mathrm{m}}=\sqrt{\beta^{2}-k_{0}^{2} \varepsilon_{\mathrm{m}}},
$$

where $k_{0}$ represents the wave number of light in free space. The temporal coupled-mode theory (CMT) is used to account for the dynamic transmission characteristics. For the resonator-coupled waveguide system in Figure 1, the coupling coefficient between waveguide and stub $1(\operatorname{stub} 2)$ are $\kappa_{\mathrm{w} 1}\left(\kappa_{\mathrm{w} 2}\right)$. The coupling coefficient between disk resonator and stub 1 is denoted by $\kappa_{1 \mathrm{~d}}$. The decay rates due to the internal loss in the cavities are $\kappa_{1}, \kappa_{2}$ and $\kappa_{\mathrm{d}}$, which can be neglected. The amplitudes of the incoming and outgoing waves in in waveguide are denoted by $S_{i+}$ and $S_{i^{-}}(i=1,2)$.

Considering a waveguide structure which is composed of a stub coupled with a disk cavity, the normalized amplitude $a_{1}$ of the stub and $a_{\mathrm{d}}$ of the disk can be expressed as follows: 


$$
\begin{aligned}
\frac{\mathrm{d} a_{1}}{\mathrm{~d} t}= & \left(j \omega_{1}-\kappa_{\mathrm{w} 1}-\kappa_{1 \mathrm{~d}}-\kappa_{1}\right) a_{1} \\
& +j \sqrt{\kappa_{\mathrm{w} 1}} S_{1+}+j \sqrt{\kappa_{\mathrm{w} 1}} S_{2+}+j \sqrt{\kappa_{1 \mathrm{~d}}} a_{\mathrm{d}}, \\
& \frac{\mathrm{d} a_{\mathrm{d}}}{\mathrm{d} t}=\left(j \omega_{\mathrm{d}}-\kappa_{1 \mathrm{~d}}\right) a_{\mathrm{d}}+j \sqrt{\kappa_{1 \mathrm{~d}}} .
\end{aligned}
$$

According to energy conservation, the amplitude of the incoming and the outgoing waves in coupled waveguides should satisfy the following relationships:

$$
\begin{aligned}
& S_{2+}=S_{1+}+j \sqrt{\kappa_{\mathrm{w} 1}} a_{1}, \\
& S_{1-}=S_{2-}+j \sqrt{\kappa_{\mathrm{w} 1}} a_{1} .
\end{aligned}
$$

When an optical wave with frequency $\omega$ is launched only from the input port of the bus waveguide, $S_{2+}=0$. The transmission and reflection coefficients of the stub-disk system can be derived as:

$$
\begin{aligned}
t_{1 \mathrm{~d}} & =\frac{S_{2-}}{S_{1+}} \\
& =\frac{j\left(\omega-\omega_{1}\right)+\kappa_{1 \mathrm{~d}}+\kappa_{1}+\frac{\kappa_{1 \mathrm{~d}}}{j\left(\omega-\omega_{\mathrm{d}}\right)+\kappa_{1 \mathrm{~d}}}}{j\left(\omega-\omega_{1}\right)+\kappa_{\mathrm{w} 1}+\kappa_{1 \mathrm{~d}}+\kappa_{1}+\frac{\kappa_{1 \mathrm{~d}}}{j\left(\omega-\omega_{\mathrm{d}}\right)+\kappa_{1 \mathrm{~d}}}} \\
& =1-\frac{\kappa_{w 1}}{\beta_{1}+\frac{\kappa_{1 d}}{\beta_{d}}}, \\
r_{1 \mathrm{~d}} & =\frac{S_{1-}}{S_{1+}} \\
& =-\frac{\kappa_{\mathrm{w} 1}}{j\left(\omega-\omega_{1}\right)+\kappa_{\mathrm{w} 1}+\kappa_{1 \mathrm{~d}}+\frac{\kappa_{1 \mathrm{~d}}}{j\left(\omega-\omega_{1 \mathrm{~d}}\right)+\kappa_{1 \mathrm{~d}}}} \\
& =-\frac{\kappa_{\mathrm{w} 1}}{\beta_{1}+\frac{\kappa_{1 \mathrm{~d}}}{\beta_{\mathrm{d}}}}
\end{aligned}
$$

where $\beta_{1}=j\left(\omega-\omega_{1}\right)+\kappa_{\mathrm{w} 1}+\kappa_{1 \mathrm{~d}}+\kappa_{1}$, and $\beta_{\mathrm{d}}=j\left(\omega-\omega_{1 \mathrm{~d}}\right)+$ $\kappa_{1 \mathrm{~d}}$. From Equation 5, the transmission efficiency of stub-disk coupled system can be expressed as:

$$
T=\left|t_{1 \mathrm{~d}}\right|^{2}=\left|1-\frac{\kappa_{\mathrm{w} 1}}{\beta_{1}+\frac{\kappa_{1 \mathrm{~d}}}{\beta_{\mathrm{d}}}}\right|^{2}
$$

Neglecting the internal loss $\kappa_{1}$, we find the transmission response of stub-disk system to be

$$
T=\left|t_{1 \mathrm{~d}}\right|^{2}=\left|\frac{j\left(\omega-\omega_{1}\right)+\kappa_{1 \mathrm{~d}}+\frac{\kappa_{1 \mathrm{~d}}}{j\left(\omega-\omega_{\mathrm{d}}\right)+\kappa_{1 \mathrm{~d}}}}{j\left(\omega-\omega_{1}\right)+\kappa_{\mathrm{w} 1}+\kappa_{1 \mathrm{~d}}+\frac{\kappa_{1 \mathrm{~d}}}{j\left(\omega-\omega_{\mathrm{d}}\right)+\kappa_{1 \mathrm{~d}}}}\right|^{2} .
$$

If there is no disk resonator coupled to the stub, which means there is only one stub resonator, then $\kappa_{1 \mathrm{~d}}=0$, and Equation 5 and Equation 6 are simplified. Transmission and reflection coefficients of a waveguide with one stub are written as:

$$
\begin{gathered}
t_{i}=\frac{j\left(\omega-\omega_{i}\right)+\kappa_{i}}{j\left(\omega-\omega_{i}\right)+\kappa_{\mathrm{w} 1}+\kappa_{i}}=1-\frac{\kappa_{\mathrm{w} i}}{\beta_{i}}, \\
r_{i}=-\frac{\kappa_{\mathrm{w} i}}{j\left(\omega-\omega_{i}\right)+\kappa_{\mathrm{w} i}+\kappa_{i}}=-\frac{\kappa_{\mathrm{w} i}}{\beta_{i}} .
\end{gathered}
$$

The subscripts $i=1,2$ stand for stub 1 and stub 2, respectively; $\beta_{2}=j\left(\omega-\omega_{2}\right)+\kappa_{\mathrm{w} 2}+\kappa_{2}$. Through the use of Equation 9, the transmission efficiency of an individual resonator stub is given by:

$$
T=\left|t_{i}\right|^{2}=\left|1-\frac{\kappa_{\mathrm{w} i}}{\beta_{i}}\right|^{2}=\frac{\left(\omega-\omega_{i}\right)^{2}+\kappa_{i}^{2}}{\left(\omega-\omega_{i}\right)^{2}+\left(\kappa_{\mathrm{w} i}+\kappa_{i}\right)^{2}} .
$$

Equation 11 holds for any individually coupled resonator whether it is a stub or a disk resonator. Therefore Equation 11 presents the general expression of transmission response for an individual resonator. In this paper, the subscripts $i=1,2, \mathrm{~d}$ stand for stub 1, stub 2 and the disk resonator, respectively. Equation 11 is consistent with the result in [29].

For a waveguide system coupled to multiple resonators, the incident and output waves through the entire system show a transfer characteristic. The transmission is determined by a transfer matrix [30]. We regard that the structure shown in Figure 1 is composed of two resonator subsystems. The first subsystem consists of stub 1 and disk, and the second subsystem is only stub 2 . Then the transmission of the entire system is described by: 


$$
T=\left|\frac{t_{1 \mathrm{~d}} t_{2}}{1-r_{1 \mathrm{~d}} r_{2} e^{j \psi}}\right|^{2},
$$

where $t_{1 \mathrm{~d}}\left(r_{1 \mathrm{~d}}\right)$ and $t_{2}\left(r_{2}\right)$ denote the transmission (reflection) coefficients of 1 st and 2 nd resonator subsystem. $\psi$ represents the phase difference between the subsystems. In our case the separation distance between two stubs is zero, $\psi=0$, so the transmission is written as:

$$
T=\left|\frac{\left(1-\frac{\kappa_{\mathrm{w} 1}}{\beta_{1}+\frac{\kappa_{1 \mathrm{~d}}}{\beta_{\mathrm{d}}}}\right)\left(1-\frac{\kappa_{\mathrm{w} 2}}{\beta_{2}}\right)}{1-\left(\frac{\kappa_{\mathrm{w} 1}}{\beta_{1}+\frac{\kappa_{1 \mathrm{~d}}}{\beta_{\mathrm{d}}}}\right)\left(\frac{\kappa_{\mathrm{w} 2}}{\beta_{2}}\right)}\right|^{2} .
$$

From Equation 13 the transmission of entire system is obtained. The results for simple resonator structures can be derived based on Equation 13. If there is no stub 2, $\kappa_{\mathrm{w} 2}=0$, then Equation 13 reduces to

$$
T=\left|1-\frac{\kappa_{\mathrm{w} 1}}{\beta_{1}+\frac{\kappa_{1 \mathrm{~d}}}{\beta_{\mathrm{d}}}}\right|^{2},
$$

which is the same as Equation 7. It describes the transmission efficiency of the stub-disk coupled system. If there is no disk, $\kappa_{1 \mathrm{~d}}=0$, then Equation 13 is simplified to Equation 14 (see below).

This is the transmission efficiency of a two-side coupled-stubs structure. Assume the two stubs are identical, then $\kappa_{\mathrm{w} 1}=\kappa_{\mathrm{w} 2}=$ $\kappa_{\mathrm{w}}, \beta_{1}=\beta_{2}=\beta_{0}$ and $\omega_{1}=\omega_{2}=\omega_{0}$. Ignoring the loss, Equation 14 can be derived as:

$$
T=\left|\frac{j\left(\omega-\omega_{0}\right)}{j\left(\omega-\omega_{0}\right)+2 \kappa_{\mathrm{w}}}\right|^{2}=\frac{\left(\omega-\omega_{0}\right)^{2}}{\left(\omega-\omega_{i}\right)^{2}+4 \kappa_{\mathrm{w}}{ }^{2}}
$$

Thus, the transmission efficiency of two-side coupled cavities is obtained, which is consistent with the results in [31]. If there is no disk and no stub 2, $\kappa_{1 \mathrm{~d}}=0$ and $\kappa_{\mathrm{w} 2}=0$, then Equaiton 13 reduces to

$$
T=\left|1-\frac{\kappa_{\mathrm{w} 1}}{\beta_{1}}\right|^{2}
$$

which is same as Equation 11. This is exactly the transmission efficiency of a one-stub system.

\section{Results and Discussion}

Numerical simulations were performed by using COMSOL Multiphysics to investigate the spectral response. In the simulations, we fix $W=50 \mathrm{~nm}$ and $g=10 \mathrm{~nm}$. The radius of the disk is $R=280 \mathrm{~nm}$. The widths of stubs are $W_{1}=100 \mathrm{~nm}$, and $W_{2}=100 \mathrm{~nm}$. The lengths of the stubs are $L_{1}=180 \mathrm{~nm}$, and $L_{2}=280 \mathrm{~nm}$.

The obtained transmission spectra for different resonatorcoupled waveguide structures are plotted in Figure 2. It is seen that for the individual resonator, there is a wavelength range in which the transmittance is low, as shown by the green and blue lines. For the stub-disk coupled waveguide structure, a transmission peak appears in the transmission dip, as shown by the red line in Figure 2a. For two-stub coupled structures, a transmission peak is found, as shown by red line in Figure $2 \mathrm{~b}$. This phenomenon is similar to electromagnetically induced transparency (EIT) [32,33].

The above transmission results can be quantitatively explained by our theory model. Equation 12 demonstrates that the minimum transmission $T_{\min }$ occurs when $\omega_{i}=\omega$. Then, $T_{i, \min }=\left|\kappa_{i} /\left(\kappa_{\mathrm{w} i}+\kappa_{i}\right)\right|^{2}$. Neglecting the internal loss $\kappa_{i}$, we have $T_{\min }=0$. This means that a wave with resonance frequency $\omega_{i}$ will be suppressed and not transmitted. Based on Equation 11, one can explain the transmission dip around the resonance frequencies $\omega_{1}, \omega_{2}$ and $\omega_{\mathrm{d}}$ for the individual resonators stub 1 and stub 2, and the disk, respectively.

Note that the individual stub resonator has very broad transmission dip. The broad dip is caused by the direct coupling. Because stub resonators are directly connected to the MDM

$$
T=\left|\frac{\left(1-\frac{\kappa_{\mathrm{w} 1}}{\beta_{1}}\right)\left(1-\frac{\kappa_{\mathrm{w} 2}}{\beta_{2}}\right)}{1-\left(\frac{\kappa_{\mathrm{w} 1}}{\beta_{1}}\right)\left(\frac{\kappa_{\mathrm{w} 2}}{\beta_{2}}\right)}\right|^{2}=\left|\frac{\left(1-\frac{\kappa_{\mathrm{w} 1}}{j\left(\omega-\omega_{1}\right)+\kappa_{\mathrm{w} 1}+\kappa_{1 \mathrm{~d}}+\kappa_{1}}\right)\left(1-\frac{\kappa_{\mathrm{w} 2}}{j\left(\omega-\omega_{2}\right)+\kappa_{\mathrm{w} 2}+\kappa_{2}}\right)}{1-\left(\frac{\kappa_{\mathrm{w} 1}}{j\left(\omega-\omega_{1}\right)+\kappa_{\mathrm{w} 1}+\kappa_{1 \mathrm{~d}}+\kappa_{1}}\right)\left(\frac{\kappa_{\mathrm{w} 2}}{j\left(\omega-\omega_{2}\right)+\kappa_{\mathrm{w} 2}+\kappa_{2}}\right)}\right|
$$



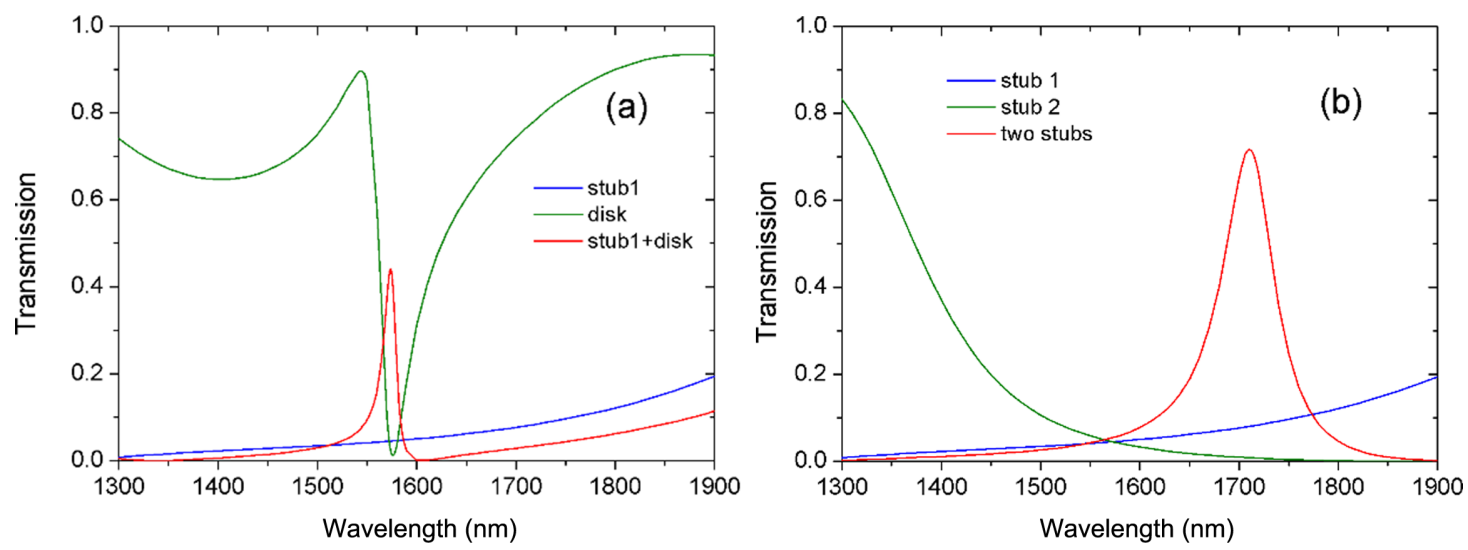

Figure 2: Transmission spectra for different resonator-coupled structures. The radius of the disk is $R=280 \mathrm{~nm}$. The lengths of the stubs are $L_{1}=180 \mathrm{~nm}, L_{2}=280 \mathrm{~nm}$. (a) The individual stub 1 resonator, individual disk resonator, and coupled stub-disk system. (b) The individual stub 1 resonator, individual stub 2 resonator, and coupled-stubs system.

waveguide, the energy stored in the stubs will be reduced and the quality factor of the stubs will decrease. As a result, broad transmission dips are formed. The disk resonator has narrower transmission dip than the stubs. This is because the disk is sidecoupled to the waveguide. Besides, the disk resonator can support whispering gallery modes. These can greatly reduce the propagation loss in the disk resonator. Therefore its quality factor is higher and the corresponding transmission dip is narrower. The spectral shape of the transmission for a single resonator agrees well with the theoretical analysis.

When a disk is coupled to the upper stub 1 , cavities modes can directly couple with each other through their evanescent field at small gap distances. When $\omega_{1}=\omega_{\mathrm{d}}=\omega$, the transmittance of Equation 8 becomes $T=\left|\left(\kappa_{1 \mathrm{~d}}+1\right) /\left(\kappa_{\mathrm{w} 1}+\kappa_{1 \mathrm{~d}}+1\right)\right|^{2}$. This indicates that a transmission peak emerges in the transmission dip. Based on Equation 8 and Equation 11, we can explain the EITlike phenomenon of the transmission peak in Figure 2.
Figure 3 shows the transmission spectrum for resonator-coupled waveguide structures in which two transmission peaks appear. The corresponding peak wavelengths are $\lambda_{\mathrm{I}}=1557 \mathrm{~nm}$ and $\lambda_{\mathrm{II}}=1791 \mathrm{~nm}$. This phenomenon can also be explained by our theory. Equation 13 indicates that there would be a transmission peak at frequency $\omega_{\mathrm{d}}=\omega$ as long as $\omega_{1}$ and $\omega_{\mathrm{d}}$ are close to each other, and another peak at frequency $\omega_{2}=\omega$, as long as $\omega_{1}$ and $\omega_{2}$ are close to each other. According to this analysis, we know there are two transmission peaks. Peak I is near the resonance frequency of the disk $\left(\omega_{\mathrm{d}}\right)$, and peak II is around the resonance frequency of stub $2\left(\omega_{2}\right)$. The obtained spectra agree well with the theoretical prediction.

To further reveal the origin of the transmission peaks in Figure $3 \mathrm{a}$, we plot the distribution of the magnetic field $H_{z}$ at the two resonance wavelengths in Figure $3 \mathrm{~b}$. It is seen that the energy is mostly confined in the disk resonator at the frequency of resonance peak I. In contrast, at the frequency of resonance

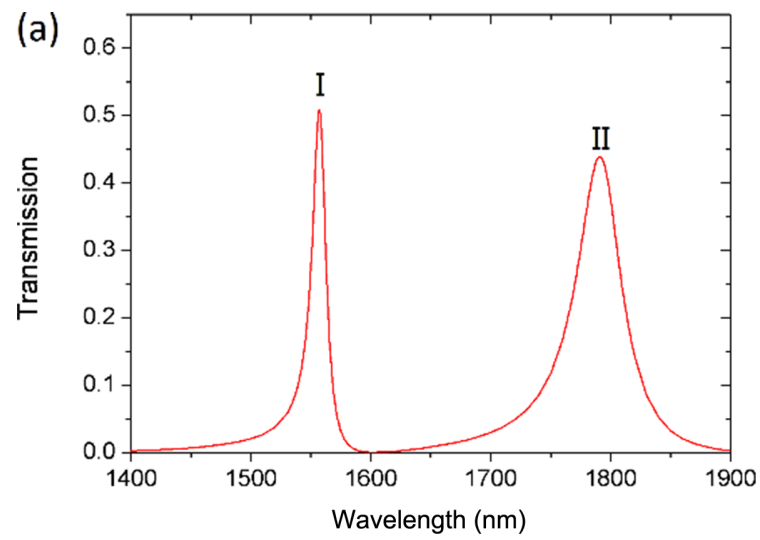

(b)
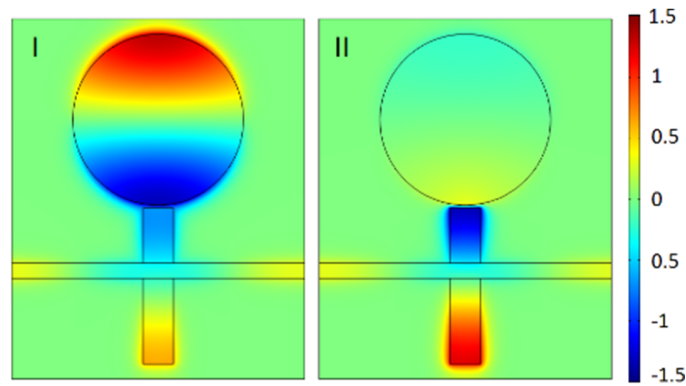

Figure 3: (a) Transmission spectra of the entire structure. (b) $H_{z}$ field patterns at the peak wavelengths of the two resonances. The radius of the disk is $R=280 \mathrm{~nm}$. The lengths of the stubs are $L_{1}=180 \mathrm{~nm}, L_{2}=280 \mathrm{~nm}$. 
peak II the energy is confined in the two stubs and there is almost no energy in the disk resonator. We infer that peak I results from the coupling between disk and stub 1 and peak II is related to the resonance between stub 1 and stub 2 .

In order to verify the above inference, we investigate the transmission when changing the structural size of the resonators. Figure 4a presents the transmission as a function of the disk radius $R$. The spectra at different stub lengths and widths are plotted in Figure 4b-e. The position of transmission peak I exhibits an obvious red shift with the increase of $R$ (Figure $4 \mathrm{a}$ ). In contrast, this peak is hardly affected when the length $\left(L_{1}, L_{2}\right)$ or the width $\left(W_{1}, W_{2}\right)$ of the stubs change (Figure $\left.4 \mathrm{~b}-\mathrm{e}\right)$. The resonance wavelength of peak I is mainly determined by the disk resonator. This indicates that peak I originates from the coupling between disk and stub 1 . Figure 4 a shows that, in contrast to peak I, the position of transmission peak II remains almost unchanged with the increase of $R$. Nevertheless, peak II is
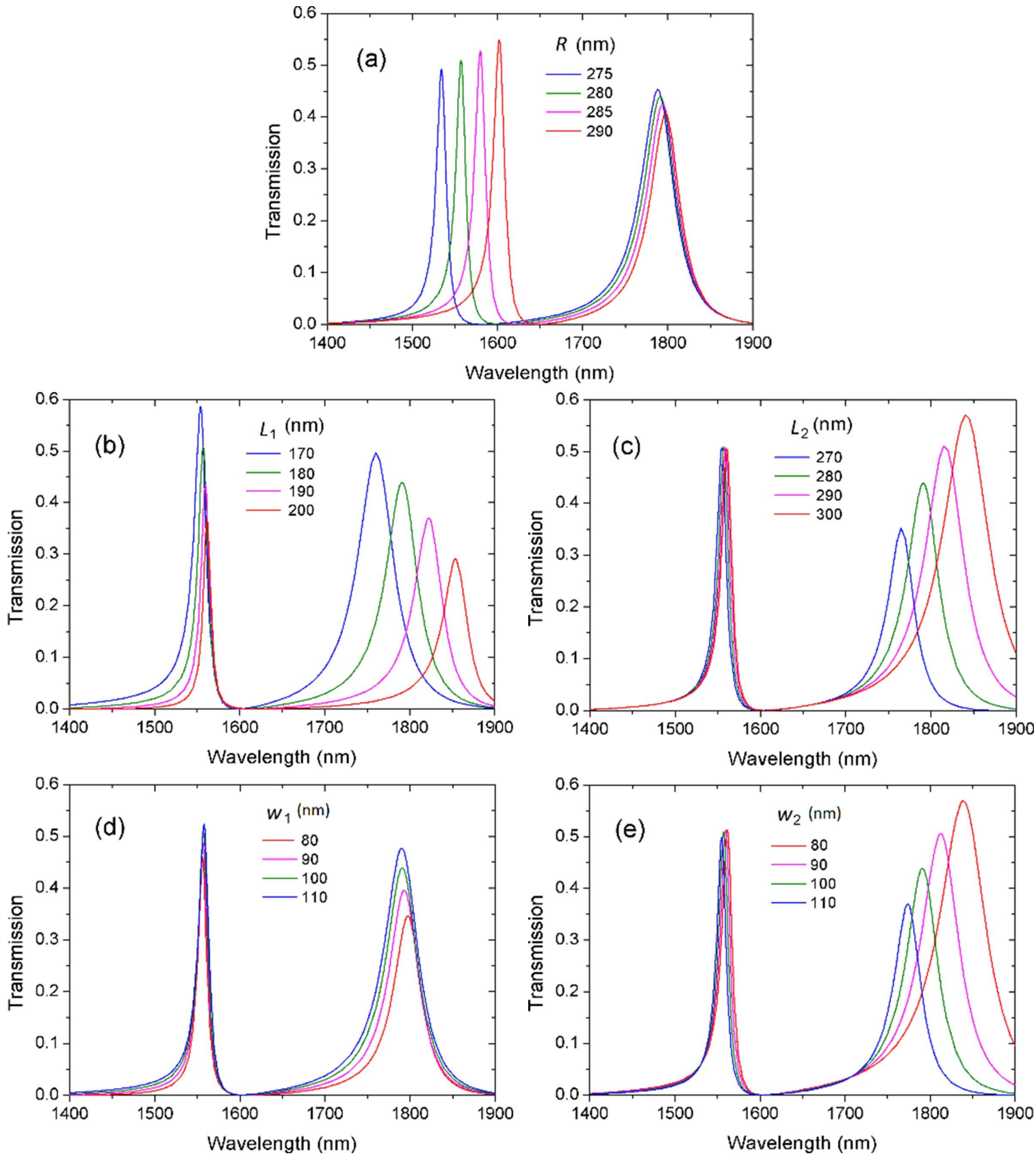

Figure 4: Transmission spectra as functions of the structural parameters: (a) radius of disk $R$; (b) length of upper stub $L_{1}$; (c) length of lower stub $L_{2}$; (d) width of upper stub $W_{1}$; (e) width of lower stub $W_{2}$. 
shifted to longer wavelengths when the length $\left(L_{1}, L_{2}\right)$ of the stubs increases or when the width $\left(W_{1}, W_{2}\right)$ of stubs decreases (Figure $4 \mathrm{~b}-\mathrm{e}$ ). The resonance wavelength of peak II is strongly influenced by the two stubs. This indicates that peak II is related to the stub 1 and stub 2 . The simulated results confirm the theoretical prediction.

Figure 4 shows that flexible modulations of the resonance wavelengths can be realized. Two resonance peaks can be easily tuned to certain wavelengths by changing specific structural parameters. This independently tunable dual resonance is promising in the application of multi-parameter sensing.

Next we investigate the effect of the refractive index on the transmission peaks. Since the left resonance peak is mainly influenced by the disk, we fill the disk cavity with a sensing medium, namely a magnetic fluid. The refractive index of the MF $\left(n_{\mathrm{MF}}\right)$ varies with temperature $T$ and external field strength $H$. The value of $n_{\mathrm{MF}}$ does not change until the field strength exceeds a critical value $H_{\mathrm{c}}$. Then, $n_{\mathrm{MF}}$ increases with rising field strengths and finally reaches a saturation value $n_{\text {sat }} \cdot n_{\mathrm{MF}}$ follows the Langevin function [2]:

$n_{\mathrm{MF}}(H, T)=\left(n_{\mathrm{sat}}-n_{0}\right)\left[\operatorname{coth}\left(\frac{H-H_{\mathrm{c}}}{T}\right)-\left(\frac{T}{\alpha\left(H-H_{\mathrm{c}}\right)}\right)+n_{0}\right]$,

for $H>H_{\mathrm{c}}$.

In Equation 15, $H$ is the magnetic field strength, $T$ represents the temperature, and $\alpha$ is a fitting parameter. $H_{\mathrm{c}}$ is the critical field (ca. $30 \mathrm{Oe}$ ). $n_{0}$ denotes the refractive index of the magnetic fluid at magnetic fields lower than the critical field. It depends on the type of carrier liquid and the concentration of the magnetic fluid. $n_{\text {sat }}$ is the saturation value of the refractive index. In this paper, the magnetic fluid is a colloidal solution consisting of $\mathrm{Fe}_{3} \mathrm{O}_{4}$ nanoparticles dispersed in water with $n_{0}=1.4612$. The concentration of the magnetic particles is $1.52 \%$ at $24.3{ }^{\circ} \mathrm{C}$. The curve of $n_{\mathrm{MF}}$ as function of $H$ becomes saturated at $H>200 \mathrm{Oe}$. The response of the refractive index to $H$ is nonlinear, but in the range of $40-100 \mathrm{Oe}$, the response shows a good linearity. Therefore field strengths of $H=40-100$ Oe are considered in this paper. $n_{\mathrm{MF}}$ increases from 1.4623 to 1.464 when the magnetic field $H$ increases from 40 to 100 Oe.

Figure 5 shows the transmission spectra as a function of the external magnetic field $H$. The left resonance at lower wavelengths is more influenced by the magnetic field strength than the right resonance at higher wavelengths. The left resonance is used for detecting and the unchanged resonance serves as a reference signal. The left peak exhibits a red shift with the increase of field strength. Because of the small change of the refractive index, $\Delta n_{\mathrm{MF}}=0.0017$, the shift of the left peak is not as large as that in Figure 4. The inset shows an enlarged view of the left peak. The detailed data show that the peak wavelength increases linearly with the magnetic field. Therefore, the magnetic field strength is known by measuring the resonance wavelength shift.

The sensitivity of an refraction-index sensor is usually defined as the shift in the resonance wavelength per unit variation of refractive index (nm/RIU) [34,35]. The obtained sensitivity for the left peak is $946 \mathrm{~nm} / \mathrm{RIU}$, which is comparable to other results [36-39]. For a magnetic sensor, the sensitivity is defined as the wavelength shift per unit variation of magnetic field

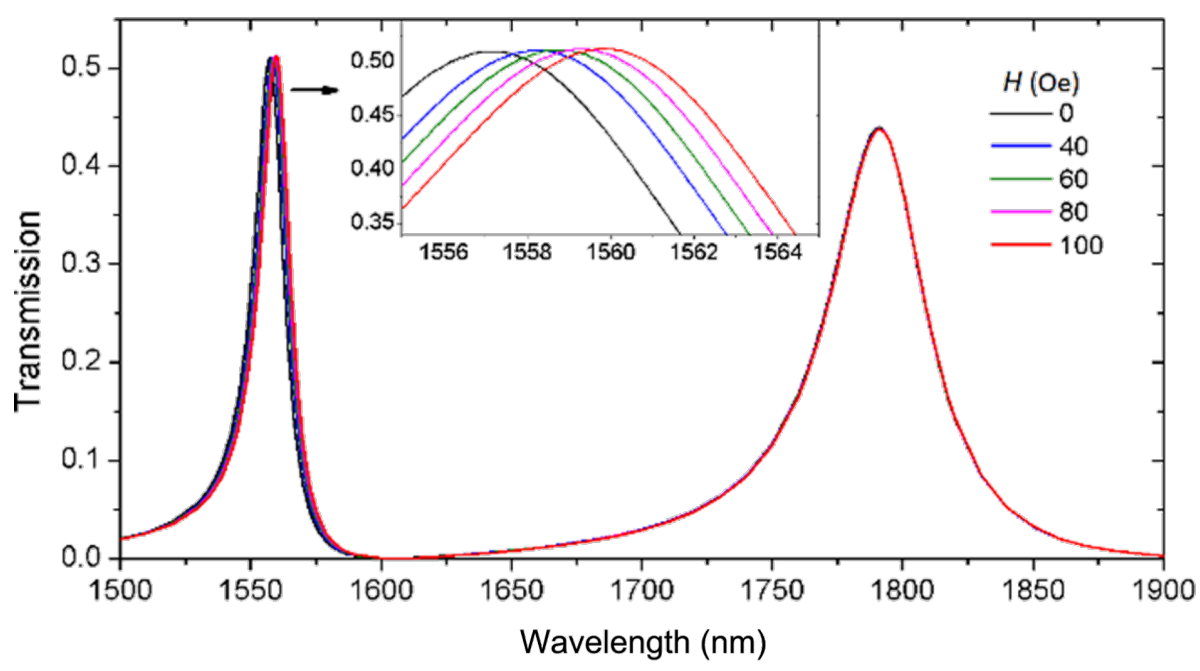

Figure 5: Transmission spectra as a function of the external magnetic field $H$. The parameters are same as in Figure 3 with the exception of the magnetic field strength. 
strength (pm/Oe). The sensitivity obtained here is $27 \mathrm{pm} / \mathrm{Oe}$, i.e., $270 \mathrm{pm} / \mathrm{mT}$, which is excellent compared to other reported values [12-15]. Because the right resonance is not sensitive to a change of the external magnetic field, it can be used as a reference. This is the characteristic of a self-reference sensor $[37,40]$. Thus, a self-reference magnetic -field sensor is achieved and the detection accuracy of the sensor can be improved. Our proposed magnetic sensor can be used in unstable and complicated environments.

\section{Conclusion}

We have demonstrated dual resonances originating from different coupling components. The resonance wavelengths are independently tuned by varying specific structural parameters. By combining a magnetic fluid and a plasmonic structure, a compact sensor that can detect the change of magnetic field strengths is achieved. The sensitivity is as high as $946 \mathrm{~nm} / \mathrm{RIU}$ for the refraction-index sensor and $27 \mathrm{pm} / \mathrm{Oe}$ (or $270 \mathrm{pm} / \mathrm{mT}$ ) for the magnetic-field sensor, respectively. The sensor features low cost, ease of fabrication and high sensitivity. In particular, because the two resonance peaks exhibit a different dependence on the change in refractive index of the magnetic fluid, the magnetic-field sensor possesses a self-reference characteristic, which can improve the accuracy of sensing. The compact magnetic sensor with high-performance is promising in the area of integrated nanoscale sensing.

\section{Acknowledgements}

This work was supported by the National Natural Science Foundation of China (11104200) and Natural Science Foundation of Tianjin (18JCYBJC17000).

\section{ORCID ${ }^{\circledR}$ iDs}

Xiaobin Ren - https://orcid.org/0000-0003-3607-5859

Qun Han - https://orcid.org/0000-0002-8139-8617

\section{References}

1. Zhao, Y.; Liu, X.; Lv, R.-Q.; Zhang, Y.-N.; Wang, Q. J. Lightwave Technol. 2017, 35, 3406-3412. doi:10.1109/jlt.2016.2573288

2. Hong, C.-Y.; Horng, H. E.; Yang, S. Y. Phys. Status Solidi C 2004, 1, 1604-1609. doi:10.1002/pssc.200304388

3. Zhao, Y.; Wu, D.; Lv, R.-Q.; Ying, Y. IEEE Trans. Magn. 2014, 50, 1-5. doi:10.1109/tmag.2014.2310710

4. Liu, J.; Lawrence, E. M.; Wu, A.; Ivey, M. L.; Flores, G. A.; Javier, K.; Bibette, J.; Richard, J. Phys. Rev. Lett. 1995, 74, 2828-2831. doi:10.1103/physrevlett.74.2828

5. Shengli, P.; Shaohua, D. IEEE Photonics J. 2014, 6, 1-6. doi:10.1109/jphot.2014.2332476

6. Wang, Q.; Liu, X.; Xia, J.; Zhao, Y. IEEE Trans. Instrum. Meas. 2015, 64, 2005-2011. doi:10.1109/tim.2014.2373516
7. Philip, J.; Jaykumar, T.; Kalyanasundaram, P.; Raj, B. Meas. Sci. Technol. 2003, 14, 1289-1294. doi:10.1088/0957-0233/14/8/314

8. Dai, Q.-F.; Deng, H.-D.; Zhao, W.-R.; Liu, J.; Wu, L.-J.; Lan, S.; Gopal, A. V. Opt. Lett. 2010, 35, 97-99. doi:10.1364/ol.35.000097

9. Zu, P.; Chan, C. C.; Wen, S. L.; Jin, Y.; Liew, H. F.; Chen, L. H. IEEE Photonics J. 2012, 4, 1140-1146. doi:10.1109/jphot.2012.2205233

10. Chen, Y. F.; Han, Q.; Liu, T. G.; Lan, X. W.; Xiao, H. Opt. Lett. 2013, 38, 3999. doi:10.1364/ol.38.003999

11. Lin, W.; Miao, Y.; Zhang, H.; Liu, B.; Liu, Y.; Song, B. Appl. Phys. Lett. 2013, 103, 151101. doi:10.1063/1.4824470

12. Zu, P.; Chan, C. C.; Lew, W. S.; Jin, Y.; Zhang, Y.; Liew, H. F.; Chen, L. H.; Wong, W. C.; Dong, X. Opt. Lett. 2012, 37, 398-400. doi:10.1364/ol.37.000398

13. Zhao, Y.; Wu, D.; Lv, R.-Q. IEEE Photonics Technol. Lett. 2015, 27, 26-29. doi:10.1109/lpt.2014.2360531

14. Miao, Y.; Ma, X.; Wu, J.; Song, B.; Zhang, H.; Zhang, K.; Liu, B.; Yao, J. Opt. Lett. 2015, 40, 3905. doi:10.1364/ol.40.003905

15. Gao, R.; Lu, D.-F.; Cheng, J.; Jiang, Y.; Jiang, L.; Ye, J. S.; Qi, Z.-M. J. Lightwave Technol. 2016, 34, 3490-3495. doi:10.1109/jt.2016.2580566

16. Gramotnev, D. K.; Bozhevolnyi, S. I. Nat. Photonics 2010, 4, 83-91. doi:10.1038/nphoton.2009.282

17. Zhang, Z.; Shi, F. H.; Chen, Y. H. Plasmonics 2015, 10, 139-144. doi:10.1007/s11468-014-9787-z

18. Piao, X.; Yu, S.; Park, N. Opt. Express 2012, 20, 18994-18999. doi:10.1364/oe.20.018994

19. Gosciniak, J.; Markey, L.; Dereux, A.; Bozhevolnyi, S. I. Nanotechnology 2012, 23, 444008. doi:10.1088/0957-4484/23/44/444008

20. Nurmohammadi, T.; Abbasian, K.; Yadipour, R. Opt. Commun. 2018, 410, 142-147. doi:10.1016/j.optcom.2017.09.082

21. Lu, H.; Liu, X.; Mao, D.; Wang, G. Opt. Lett. 2012, 37, 3780-3782. doi:10.1364/ol.37.003780

22. Yun, B.; Hu, G.; Zhang, R.; Cui, Y. Opt. Express 2014, 22, 28662-28670. doi:10.1364/oe.22.028662

23. Fan, C.; Shi, F.; Wu, H.; Chen, Y. Opt. Lett. 2015, 40, 2449-2452. doi:10.1364/ol.40.002449

24. Chen, J.; Li, Z.; Zou, Y.; Deng, Z.; Xiao, J.; Gong, Q. Plasmonics 2013, 8, 1627-1631. doi:10.1007/s11468-013-9580-4

25. Chen, Z.; Cao, X.; Song, X.; Wang, L.; Yu, L. Plasmonics 2016, 11 , 307-313. doi:10.1007/s11468-015-0035-y

26. Li, B.-X.; Li, H.-J.; Zeng, L.-L.; Zhan, S.-P.; He, Z.-H.; Chen, Z.-Q.; Xu, H. J. Lightwave Technol. 2016, 34, 3342-3347. doi:10.1109/jlt.2016.2572723

27. Wen, K.; Hu, Y.; Chen, L.; Zhou, J.; He, M.; Lei, L.; Meng, Z. Plasmonics 2017, 12, 427-431. doi:10.1007/s11468-016-0281-7

28. Dionne, J. A.; Sweatlock, L. A.; Atwater, H. A.; Polman, A. Phys. Rev. B 2006, 73, 035407. doi:10.1103/physrevb.73.035407

29. Tang, B.; Dai, L.; Jiang, C. Phys. Lett. A 2012, 376, 1234-1238. doi:10.1016/j.physleta.2012.02.009

30. Lu, H.; Liu, X. M.; Mao, D. Phys. Rev. A 2012, 85, 053803 doi:10.1103/physreva.85.053803

31. Xu, L.; Wang, S.; Wu, L. IEEE Trans. Nanotechnol. 2014, 13, 875-880. doi:10.1109/tnano.2014.2346392

32. Zhang, S.; Genov, D. A.; Wang, Y.; Liu, M.; Zhang, X. Phys. Rev. Lett. 2008, 101, 047401. doi:10.1103/physrevlett.101.047401

33. Liu, N.; Langguth, L.; Weiss, T.; Kästel, J.; Fleischhauer, M.; Pfau, T.; Giessen, H. Nat. Mater. 2009, 8, 758-762. doi:10.1038/nmat2495 
34. Mayer, K. M.; Hafner, J. H. Chem. Rev. 2011, 111, 3828-3857. doi:10.1021/cr100313v

35. Ren, X.; Ren, K.; Cai, Y. Appl. Opt. 2017, 56, H1-H9. doi:10.1364/ao.56.0000h1

36. Shahamat, Y.; Vahedi, M. Opt. Commun. 2017, 401, 40-45. doi:10.1016/j.optcom.2017.05.016

37. Wang, Y.; Sun, C.; Li, H.; Gong, Q.; Chen, J. Nanoscale 2017, 9, 11085-11092. doi:10.1039/c7nr04259k

38. Lai, W.; Wen, K.; Lin, J.; Guo, Z.; Hu, Q.; Fang, Y. Appl. Opt. 2018, 57 6369-6374. doi:10.1364/ao.57.006369

39. Wang, Q.; Ouyang, Z.; Sun, Y.; Lin, M.; Liu, Q.; Zheng, G.; Fan, J. Sensors 2018, 18, 1026. doi:10.3390/s18041026

40. Ren, X.; Ren, K.; Ming, C. Sensors 2018, 18, 1376. doi:10.3390/s18051376

\section{License and Terms}

This is an Open Access article under the terms of the Creative Commons Attribution License (http://creativecommons.org/licenses/by/4.0). Please note that the reuse, redistribution and reproduction in particular requires that the authors and source are credited.

The license is subject to the Beilstein Journal of Nanotechnology terms and conditions: (https://www.beilstein-journals.org/bjnano)

The definitive version of this article is the electronic one which can be found at: doi:10.3762/bjnano. 10.23 